\title{
Diseño y validación de un instrumento para medir los conocimientos en matemáticas básica que poseen los estudiantes de ciencias
}

\author{
Design and validation of an instrument to measure knowledge in basic \\ mathematics that science students possess \\ Francisco de Asís Carreras García. ${ }^{1}$ \& Zoraida Margarita Sívoli Barrios. ${ }^{2}$ \\ Recibido: 16-11-2019 / Revisado: 1-12-2019/Aceptado: 25-12-2019/ Publicado: 04-01-2020
}

\begin{abstract}
DOI: https://doi.org/10.33262/cienciadigital.v4i1.1098

The purpose of this article is to develop and validate an instrument designed to measure knowledge in basic mathematics. Pointing the importance of validation as an orderly process that should lead to the reliability of the instrument. The design has been structured in 8 steps, initially a consultation with math teachers to structure the areas that would be evaluated, after that the development of a preliminary instrument, and then validate the experts and finally be able to develop the desired assessment instrument. The construction has been developed in the areas of basic mathematics: Arithmetic, Algebra, Trigonometry and Geometry, which were subdivided into sub-areas such as order of numbers, factorization, trigonometric ratios in a right triangle, calculate the area of geometric figures, between others, because they are the ones that mathematics teachers considered most necessary for the first courses of the Science careers. A pilot test was carried out on a group of students of Biochemistry in the first semester and the result of the Cronbach Alpha coefficient obtained determines a good indicator of reliability. This allows us to consider that the instrument is well constructed and can be for the group for which the design of the instrument was raised. Keywords: Basic knowledge, Mathematics, Assessment instrument, Science students.

\section{Resumen}

El propósito de este artículo es desarrollar y validar un instrumento diseñado para medir conocimientos en matemáticas básica. Señalando la importancia de la validación como un
\end{abstract}

1 Escuela Politécnica de Chimborazo, Facultad de Ciencias, Escuela de Física y Matemática, francisco.carreras@espoch.edu.ec

2 Escuela Politécnica de Chimborazo, Facultad de Ciencias, Escuela de Física y Matemática, zoraida.sivoli@espoch.edu.ec 
proceso ordenado que debe conducir a la confiabilidad del instrumento. El diseño se ha estructurado en 8 pasos, inicialmente una consulta a docentes de matemáticas para estructurar las áreas que serían evaluadas, luego el desarrollo de un instrumento preliminar, para después hacer la validación de los expertos y finalmente poder elaborar el instrumento de evaluación deseado. La construcción se ha elaborado en las áreas de matemática básica: Aritmética, Algebra, Trigonometría y Geometría, las cuales se subdividieron en subáreas como orden de los números, factorización, razones trigonométricas en un triángulo rectángulo, calcular el área de las figuras geométricas, entre otros, por ser las que los docentes de matemáticas consideraban más necesarias para los primeros cursos de las carreras de Ciencias. Se realizó una prueba piloto a un grupo de estudiantes de Bioquímica del primer semestre y el resultado del coeficiente de Alfa de Cronbach obtenido determina un buen indicador de confiabilidad. Esto nos permite considerar que el instrumento está bien elaborado y puede ser $\mathrm{p}$ al grupo para el cual se planteó el diseño del mismo.

Palabras claves: Conocimientos Básicos, Matemática, Instrumento de evaluación, Estudiantes de ciencias.

\section{Introducción}

El objetivo de este estudio es elaborar un instrumento de medición, válido y fiable, que permita determinar el nivel de conocimientos en matemática básica de los estudiantes que ingresan a estudiar en la Facultad de Ciencias de la Escuela Superior Politécnica de Chimborazo (ESPOCH) del Ecuador, como el paso inicial que debe conocer el docente cuando va a impartir un curso de matemática del primer semestre.

En el proceso de aprendizaje de la matemática, el estudiante recibe continuos estímulos asociados a esta área del conocimiento (problemas, actuaciones del profesor, mensajes sociales) que los llevan a reaccionar emocionalmente de forma positiva o negativa según Muñoz (2006), estos estímulos de alguna forma condicionan su actitud hacia las matemáticas y por ende la asimilación de los conocimientos que el recibe. Sin embargo, cuando se necesita conocimientos para el estudio de una carrera del área científica esos estímulos no pueden ser considerados para el ingreso del estudiante porque la necesidad de los conocimientos está por encima de su posible predisposición a la matemática.

En la Facultad de Ciencias de la Escuela Superior Politécnica de Chimborazo (en Ecuador) se imparten las carreras de Matemática, Física, Química, Estadística, Ingeniería Química, Ingeniería 
Ambiental y Bioquímica y Farmacia, las cuales están enfocadas en la formación de los estudiantes con una gran base científica y tecnológica para poder ingresar en el campo de la investigación y así brindar apoyo y asesoría a las empresas públicas y privadas de la región y el país.

Para este fin, la necesidad de que el estudiante tenga una base sólida de conocimientos en matemáticas al ingresar a estudiar dichas carreras es una necesidad ineludible, por lo cual el conocer con qué formación en matemática básica (aritmética, algebra, geometría y trigonometría) inician los estudiantes es una preocupación dentro de la planta profesoral.

Esta preocupación y los estándares de contenido (números y operaciones, algebra, geometría y medida) que señala el Consejo Nacional de Profesores de Matemáticas de Estados Unidos, que publicó los Principios y Estándares para la Educación Matemática, National Council of Teachers of Mathematics (NTCM, 2000) nos motivó a diseñar un instrumento de evaluación que nos permitiera medir el nivel en matemáticas básica que tienen los estudiantes al ingresar a las carreras de la Facultad de Ciencias.

Es de hacer notar que existen algunos instrumentos de medición de actitudes y conocimientos matemáticos, ya validados, como Alemany y Lara (2010), Muñoz y Mato (2006) y Vallejo y Escudero (1999), que miden la actitud hacia la matemática de los estudiantes de la Educación Secundaria Obligatoria (ESO) en España o los que miden los procesos matemáticos en la Educación Infantil, (Alsina y Coronata 2014), o los que utilizan la versión española del Utrecht Early Numeracy Test, (Cerda et al 2012), sin embargo, al momento de hacer este estudio no encontramos ningún instrumento que mida los conocimientos en matemática básica en estudiantes que desean estudiar carreras de Ciencias en el Ecuador.

En este trabajo pretendemos construir un instrumento de evaluación apropiado, que según Alvarado, Canales y Pineda (1994) “... es el mecanismo que utiliza el investigador para recolectar y registrar la información”, el cual permita medir los conocimientos de matemática básica bajo dos aspectos fundamentales: el aprendizaje que reciben en el bachillerato y la influencia de esos temas en el ámbito universitario. De esta forma el instrumento de evaluación permite determinar las deficiencias del estudiante que hay que mejorar y las acciones que permitan elevar la calidad de los conocimientos.

En el ambiente educativo, Obando (2009) señala que los docentes de forma un tanto empírica construyen sus propias pruebas de evaluación, que son aplicadas, pero nunca son sometidas a procesos de validación y estandarización que permitan hacer inferencias de carácter sólido y 
científico. De esta forma para dar una mayor rigurosidad debemos proceder con los mecanismos que nos permitan generar confianza en los resultados que obtenemos al aplicar el instrumento o pruebas de evaluación.

La problemática al momento de la recolección de datos en un trabajo de investigación se centra en la construcción de los instrumentos a emplear con esta finalidad, de manera que permitan recabar información válida y confiable según Corral (2009). Por este motivo la confiabilidad del instrumento es lo que le da valor al estudio que se quiere realizar y permite su posterior implementación.

En el presente estudio queremos llevar a cabo la validación de un instrumento de evaluación en el área de matemáticas por considerar que es fundamental para los estudios de las carreras de Ciencias y a su vez, para que el mismo sirva de modelo metodológico en estudios similares en diferentes áreas de la educación superior universitaria, o en otras regiones geográficas.

Según Obando (2009) en el proceso de construcción de una prueba o instrumento de evaluación se contemplan las fases de: conceptualización, construcción de reactivos, validación y análisis de confiabilidad y por último la estandarización.

En la primera parte de conceptualización se determina el objetivo del estudio, a que población se le aplicará y cuál es el constructo o contenido específico a medir. En la construcción de los reactivos se debe observar si los mismos están ajustados al contenido del marco teórico de la investigación. La validación y confiablidad se puede realizar internamente o por medio de un juicio de expertos según sea la característica de la investigación.

El juicio de expertos utilizado para darle validez al instrumento permite determinar la posibilidad de errores en el contenido o en la escritura del instrumento de evaluación, uno de los métodos utilizado es el conocido como "Método de agregados individuales" en donde se hace una consulta individual con los expertos, que según García Perales (2018) “... puede parecer un método limitado porque los expertos no pueden intercambiar sus opiniones...... pero esta limitación puede ser precisamente lo que se esté buscando para evitar los sesgos".

Una vez elaborado el instrumento se necesita realizar una prueba piloto, según indica Malhotra (2004) es la aplicación del instrumento a una pequeña muestra de la población para identificar y eliminar posibles problemas en la elaboración del instrumento.

Para el análisis de confiabilidad de dicha prueba aplicamos el coeficiente Alfa de Cronbach, y según señalan Oviedo y Campo (2004) un valor entre 0,7 y 0,9 indica una buena consistencia 
interna; incluso autores como Gadermann, Guhn, y Zumbo (2012) reportan que el alfa de Cronbach ha sido citada en el $76 \%$ de los casos de artículos de Ciencias Sociales para evidenciar la validez de las pruebas.

Finalmente, según el resultado del análisis del coeficiente Alfa de Cronbach se procede a estandarizar el instrumento, si es necesario, realizando los cambios en el mismo, los cuales pueden ser modificaciones, eliminaciones o incorporaciones de nuevas preguntas.

\section{Metodología}

\section{Objetivos}

El objetivo de esta investigación es el diseño de un instrumento de medición de los conocimientos en matemáticas básicas de los estudiantes del primer semestre de la Facultad de Ciencias.

\section{Diseño}

En esta investigación usamos el método de investigación empírico-analítico para establecer una correlación, en los estudiantes que ingresan a la Universidad, entre los conocimientos académicos y las variables personales.

Hemos utilizado un diseño transversal tomando los datos de diferentes grupos de estudiantes según la carrera con la cual ellos ingresan a la Universidad, en el mismo periodo de tiempo y para examinar la relación entre las variables que deseamos estudiar.

\section{Población y Participantes.}

El grupo de estudiantes sobre los cuales aplicaremos el instrumento son los de nuevo ingreso a la Facultad de Ciencias en las carreras de Física, Química, Matemática, Estadística, Ingeniería Química, Ingeniería Ambiental y Bioquímica y Farmacia, de la Escuela Superior Politécnica de Chimborazo (ESPOCH) del Ecuador.

Estos estudiantes provienen de toda la geografía del Ecuador, tanto de instituciones públicos, privados y fiscomisionados, situados en los diferentes cantones y algunos corresponden a zonas de población indígena de origen étnico quichua, la cual es la de mayor población en el país (Robles). Bajo estas circunstancias podemos decir que el resultado de la investigación nos dará una muestra representativa del nivel de conocimiento en matemática básica de los estudiantes de todo el Ecuador.

La selección de la muestra estuvo en función de los estudiantes necesarios de nuevo ingreso para realizar los estadísticos de confiabilidad y validez del instrumento. Para ello, tomamos los criterios 
de Darias (2005) citando a Comrey $(1962,1973)$ que afirma que a partir de 200 sujetos la muestra se considera adecuada.

\section{Identificación de las variables.}

De acuerdo con nuestros objetivos consideramos dos tipos de variables:

1. Variables Sociodemográficas, que constituyen las variables cualitativas y que las clasificamos en:

a) Variables Personales: nombre, sexo, edad, cédula de identidad, código, año de graduación y entorno donde vive.

b) Variable Institución Educativa: nombre de la institución, ubicación, tipo y sistema educativo.

c) Variable de la carrera: nombre de la carrera, aprobó la nivelación, desea estudiar la carrera.

2. Variables Académicas, las cuales son las variables cuantitativas que medirán los conocimientos en matemática básica y que clasificamos por área:
a) Aritmética
b) Algebra
c) Geometría
d) Trigonometría

\section{Instrumento de medida}

Debido a que no encontramos ningún instrumento que mida los conocimientos en matemática básica bajo las características de los estudiantes a los cuales estamos dirigiendo nuestra investigación. Se decidió elaborar un instrumento de evaluación propio de selección múltiple con cuatro respuestas por pregunta de las cuales tres eran distractores, seleccionamos este tipo de preguntas debido a que requieren de procesos cognitivos de orden taxonómico menor que los empleados para la elaboración de un ensayo o un portafolios, según (Biggs 2003).

Hemos elaborado el instrumento siguiendo los pasos indicados en la (Tabla 1) en donde iniciamos con la revisión bibliográfica, seguimos con la elaboración de un instrumento inicial en base a la opinión de los expertos en matemáticas y finalmente elaboramos el instrumento definitivo con la consulta a los expertos en el área y en la elaboración de instrumentos de medición. 
Tabla 1. Pasos para la elaboración del Instrumento

\begin{tabular}{|c|c|c|}
\hline $\begin{array}{l}\text { PRIMER } \\
\text { PASO }\end{array}$ & $\begin{array}{l}\text { Revisión de la bibliografía referente a instru- } \\
\text { mentos que evalúen conocimientos en mate- } \\
\text { máticas }\end{array}$ & REVISION BIBLIOGRAFICA \\
\hline $\begin{array}{l}\text { SEGUNDO } \\
\text { PASO }\end{array}$ & $\begin{array}{l}\text { Establecer los subtemas o indicadores corres- } \\
\text { pondientes a cada área según la consulta a do- } \\
\text { centes en matemáticas }\end{array}$ & \multirow{5}{*}{$\begin{array}{l}\text { ELABORACION DE LA VER- } \\
\text { SION INICIAL DEL INSTRU- } \\
\text { MENTO DESPUES DE LA CON- } \\
\text { SULTA A EXPERTOS EN MA- } \\
\text { TEMATICAS }\end{array}$} \\
\hline $\begin{array}{l}\text { TERCER } \\
\text { PASO }\end{array}$ & $\begin{array}{l}\text { Elaborar las preguntas del instrumento inicial } \\
\text { incorporando los resultados de la consulta a do- } \\
\text { centes en matemáticas }\end{array}$ & \\
\hline $\begin{array}{l}\text { CUARTO } \\
\text { PASO }\end{array}$ & $\begin{array}{l}\text { Aplicar primera prueba piloto para analizar el } \\
\text { tiempo de aplicación }\end{array}$ & \\
\hline QUINTO & Reformulación del instrumento basado en los & \\
\hline PASO & resultados de la prueba piloto & \\
\hline SEXTO PASO & $\begin{array}{l}\text { Consulta a expertos en el área y en la elabora- } \\
\text { ción de instrumentos de medición }\end{array}$ & $\begin{array}{l}\text { ELABORACION DEL INSTRU- } \\
\text { MENTO DEFINITIVO DESPUES }\end{array}$ \\
\hline $\begin{array}{l}\text { SEPTIMO } \\
\text { PASO }\end{array}$ & Elaboración del instrumento definitivo & $\begin{array}{l}\text { DE LA CONSULTA A EXPER- } \\
\text { TOS EN EL AREA Y EN LA ELA- }\end{array}$ \\
\hline $\begin{array}{l}\text { OCTAVO } \\
\text { PASO }\end{array}$ & $\begin{array}{l}\text { Aplicación de prueba piloto para verificar la } \\
\text { confiabilidad del instrumento }\end{array}$ & $\begin{array}{l}\text { BORACION DE INSTRUMEN- } \\
\text { TOS DE MEDICION }\end{array}$ \\
\hline
\end{tabular}

Elaborado por: Autores

Fuente: Proyecto

\section{Selección de los subtemas o indicadores a evaluar.}

Se elabora un cuestionario dirigido a expertos en matemáticas, que laboran en la ESPOCH, el cual contiene un listado de los subtemas o indicadores (8 Aritmética, 12 Algebra, 5 Geometría y 5 Trigonometría) que consideramos deberían saber los estudiantes, los cuales son parte de la malla del curso Introductorio de la ESPOCH y de los libros de matemática del bachillerato del Ecuador (Ministerio de Educación).

Se les preguntó a los expertos que indicaran cuáles de estos temas consideraban eran los prioritarios a integrar el instrumento de evaluación para medir los conocimientos de Matemática Básica de los estudiantes que ingresan al primer semestre de las carreras en la Facultad de Ciencias de la ESPOCH. 
Se consultaron 10 expertos, todos ellos con experiencia en el dictado de los cursos básicos de matemática y en base a la opinión de estos se seleccionaron los subtemas o indicadores para cada área según se muestra en la (Tabla 2).

Tabla 2. Áreas y Subtemas

\begin{tabular}{|c|c|c|c|c|c|}
\hline & & ARITMETICA & ALGEBRA & GEOMETRIA & TRIGONOMETRIA \\
\hline \multirow[t]{2}{*}{$\begin{array}{l}\text { SUBTE- } \\
\text { MAS }\end{array}$} & 1 & Orden de los números & $\begin{array}{l}\text { Resolver productos } \\
\text { notables }\end{array}$ & $\begin{array}{l}\text { Calcular el área de las } \\
\text { figuras geométricas }\end{array}$ & $\begin{array}{l}\text { Razones trigonométricas } \\
\text { en un triángulo rectángulo }\end{array}$ \\
\hline & 2 & Notación científica & $\begin{array}{l}\text { Competición de cua- } \\
\text { drados }\end{array}$ & $\begin{array}{l}\text { Triángulos semejan- } \\
\text { tes }\end{array}$ & $\begin{array}{l}\text { Identidades trigonométri- } \\
\text { cas }\end{array}$ \\
\hline \multirow[t]{2}{*}{$\mathbf{o}$} & 3 & $\begin{array}{l}\text { Orden jerárquico en } \\
\text { que se realizan las } \\
\text { operaciones }\end{array}$ & Regla de Ruffini & $\begin{array}{l}\text { Ecuación de una pará- } \\
\text { bola }\end{array}$ & $\begin{array}{l}\text { Equivalencia entre grados } \\
\text { y radianes }\end{array}$ \\
\hline & 4 & $\begin{array}{l}\text { Operaciones con frac- } \\
\text { ciones }\end{array}$ & $\begin{array}{l}\text { Despejar una variable } \\
\text { en una ecuación }\end{array}$ & & \\
\hline $\mathbf{I}$ & 5 & $\begin{array}{l}\text { Operaciones con raí- } \\
\text { ces de números reales }\end{array}$ & $\begin{array}{l}\text { Factorizar una ecua- } \\
\text { ción }\end{array}$ & & \\
\hline ND & 6 & & $\begin{array}{l}\text { Operaciones con po- } \\
\text { tencias }\end{array}$ & & \\
\hline $\mathbf{I}$ & 7 & & Racionalización & & \\
\hline $\begin{array}{l}\text { INDICADO- } \\
\text { RES }\end{array}$ & 8 & & $\begin{array}{l}\text { Algoritmo de la divi- } \\
\text { sión o división de po- } \\
\text { linomios }\end{array}$ & & \\
\hline & 9 & & Inecuaciones lineales & & \\
\hline
\end{tabular}

\section{Elaborado por: Autores}

Fuente: Consulta a expertos

Es de hacer notar que los expertos indicaron que el área de Algebra es donde los estudiantes requieren un número mayor de conocimientos, por ello es ésta el área que posee la de mayor cantidad de subtemas (Tabla 2). Con lo cual se seleccionaron 20 subtemas (5 Aritmética, 9 Algebra, 3 Geometría y 3 Trigonometría).

\section{Elaboración del Instrumento.}

Se elaboró un instrumento de selección múltiple con 3 preguntas por cada subtema, lo cual estableció un instrumento de 60 preguntas cada una con sus 3 distractores. Se estableció que el tiempo máximo para que el estudiante respondiera el instrumento sería de 90 minutos. 
En vista de que surgieron unas dudas con relación al tiempo decidimos hacer una primera prueba piloto a un grupo de 30 estudiantes. Al aplicar la prueba se determinó que el tiempo no era suficiente, ya que el 5\% de los estudiantes respondió todas las preguntas y el $80 \%$ solo logró responder 35 preguntas o menos.

Por el resultado de la primera prueba piloto se decidió reducir el número de preguntas a 2 por subtema o indicador, lo cual nos redujo el instrumento a 40 preguntas que distribuidas en las diferentes áreas nos constituyen las variables académicas cuantitativas a evaluar.

Se elaboró la sección de variables sociodemográficas en donde consideramos variables personales, variables de la institución educativa y variables de la carrera que desean estudiar.

\section{Evaluación de expertos.}

Se seleccionaron 5 expertos por su amplia trayectoria como docentes e investigadores: 1 en educación, 1 en estadística y 3 en matemáticas con el fin de revisar y emitir opinión de manera independiente tanto de los contenidos matemáticos, como claridad en la escritura y construcción del instrumento.

A los expertos se les envió información escrita sobre el objetivo del instrumento, la población a la cual se aplicaría el mismo y un cuestionario (Tabla 3) con los criterios para evaluar cada una de las 40 preguntas, en donde se les solicitaba considerar la relevancia de las preguntas acorde con el objetivo teórico, la claridad y coherencia en la redacción de la pregunta, el sesgo o tendencia de las respuestas de cada pregunta y el lenguaje apropiado al nivel de los estudiantes objeto de estudio. Al final se les pedía que señalaran la validez del instrumento seleccionando si era o no aplicable el mismo.

El instrumento enviado a los expertos señalaba, para cada dos preguntas, cuál era el objetivo para evaluar en las mismas y además indicaba la respuesta correcta (resaltada en amarillo). Por Ejemplo:

El objetivo de las siguientes preguntas es determinar si los estudiantes saben el orden jerárquico en el que se realizan las operaciones.

1. El resultado de realizar la siguiente operación es:
a. 17
b. 11
c. -1
d. -19 
2. Realice las operaciones indicadas
a. -1
b. 9
c. 15
d. 7

Tabla 3. Formato evaluación para los expertos

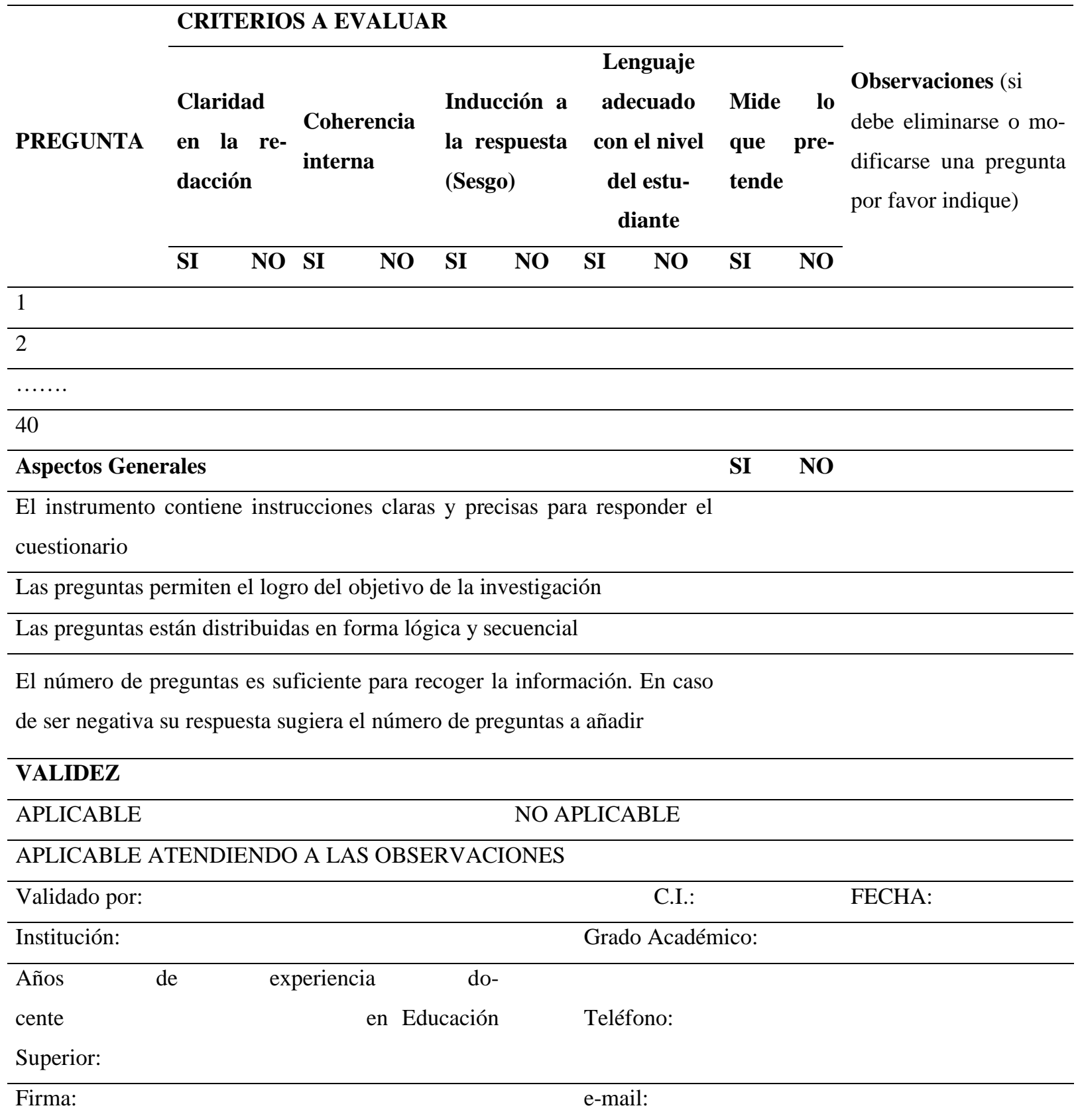

Elaborado por: Autores

Fuente: Corral, Y. (2009) 


\section{Selección de las preguntas}

Después de la evaluación de los expertos se procedió a analizar las sugerencias respectivas, las cuales iban desde mejorar la redacción de algunas de las preguntas, mantener el estilo de las preguntas, suprimir las preguntas de igual nivel de dificultad, perfeccionar alguna pregunta que estaba mal planteada y reducir el nivel de dificultad de alguna pregunta.

Se siguió el siguiente criterio para la evaluación de los expertos:

- Si había un $100 \%$ de opinión favorable de los expertos, la pregunta quedaba incluida en el instrumento.

- Si había un $80 \%$ de opinión desfavorable de los expertos, la pregunta se excluía del instrumento.

- Si había una opinión de los expertos parcial, la pregunta se revisaba, reformulaba o se sustituía

$\mathrm{Al}$ finalizar la evaluación realizada por los expertos y ajustar sus sugerencias se decidió reducir el instrumento a 32 preguntas ( 8 de Aritmética, 12 de Algebra, 6 de Geometría y 6 de Trigonometría).

\section{Validación del instrumento}

Para la validación del instrumento se realizó una prueba piloto con un grupo de 34 estudiantes del primer semestre de la carrera de Bioquímica y Farmacia de la Facultad de Ciencias de la ESPOCH. Para la confiabilidad los resultados se analizaron mediante el paquete estadístico SPSS (versión 25) calculando el coeficiente de Alpha de Cronbach.

\section{Resultados}

Se elaboró un instrumento de evaluación formado por dos secciones. La primera recoge los datos socioeconómicos del estudiante que forman parte de las variables cualitativas del instrumento:

- Datos Personales: nombre, sexo, edad, cédula de identidad, código, año de graduación y entorno donde vive.

- Datos de la Institución Educativa: nombre de la institución, ubicación, tipo y sistema educativo.

- Datos de la Carrera: nombre de la carrera, aprobó la nivelación, desea estudiar la carrera. La segunda sección recoge la información de conocimientos que poseen los estudiantes en cada una de las áreas y está conformada por 32 ítems de selección múltiple, discriminados de la siguiente manera: 
- 8 de Aritmética.

- 12 de Algebra.

- 6 de Geometría.

- 6 de Trigonometría.

La valoración que de las 32 preguntas determinará un valor para cada área en función de la cantidad de preguntas por área, lo cual medirá la cantidad de conocimientos en cada una de las áreas para contabilizar al final el rendimiento general de conocimientos matemáticos que tiene el estudiante del primer semestre de la Facultad de Ciencias, en cada una de las diferentes carreras.

Este instrumento fue validado por 5 expertos en la enseñanza en los campos de educación, estadística y matemática, los cuales tienen una experiencia docente de varios años, lo cual nos da una garantía de que el instrumento está bien elaborado y bien diseñado y sobre todo que medirá lo que realmente se quiere conocer, es decir, el conocimiento en matemática básica de los estudiantes. Después de aplicar la prueba piloto a un grupo de 34 estudiantes del primer semestre de la carrera de Bioquímica y Farmacia de la Facultad de Ciencias de la ESPOCH se obtuvo un coeficiente de 0.86 para el Alfa de Cronbach, lo cual determino que es un buen indicador de confiabilidad, Oviedo y Campo (2004).

\section{Discusión de Resultados}

Los datos socioeconómicos se incorporan con el fin de conocer la incidencia de los siguientes aspectos con el nivel de conocimiento que poseen los estudiantes:

a. El tipo de institución de la cuál egresaron, si es pública, privada o fiscomisional para determinar la diferencia en la enseñanza en cada una de esas instituciones.

b. La ubicación de la institución educativa para establecer una comparación entre las diferentes provincias de la costa, sierra y oriente.

c. El Sistema Educativo en el cuál desarrolló sus estudios de Bachiller: Sierra o Costa para poder determinar si los periodos de inicio pueden influir en los estudios de los estudiantes.

d. La edad en que realizan los estudios de su carrera por el tiempo que ha trascurrido desde que concluyó sus estudios de Bachiller para poder determinar si los conocimientos fueron afectados por el tiempo que tenía sin estudiar.

e. Si pertenece a un grupo de excelencia en sus estudios previos para ver si hay una diferencia con los estudiantes regulares. 
f. Si está motivado a seguir la carrera escogida para sus estudios universitarios para determinar si este aspecto influye en los conocimientos que debe tener para estudiar una carrera de la Facultad de Ciencias.

g. El entorno en donde vive al realizar sus estudios para ver si por ese motivo hay una deficiencia en los conocimientos mínimos para su carrera.

h. Si realizó el curso introductorio de nivelación que ofrece la Politécnica del Chimborazo para ver si se reforzaron los conocimientos necesarios para tener éxito en la carrera seleccionada.

Las preguntas se presentan en el instrumento separadas en bloques y en función de las áreas, esto se hace con el fin de que el estudiante no tenga que pasar continuamente de un nivel de razonamiento a otro al contestar la prueba, ya que las preguntas de aritmética y algebra, son netamente operativas, encontrándose categorizadas en la taxonomía de Bloom como Aplicación, mientras que en las preguntas de geometría y trigonometría se requiere Analizar los problemas para lograr su resolución.

De igual forma las preguntas se presentaron de forma aleatoria, aunque distribuidas en bloques, con un grado de dificultad media. Esta calificación se tiene en función de la cantidad de operaciones que se tengan que realizar para alcanzar el resultado de la pregunta.

Los distractores eran tres y fueron elaborados de manera que se llegase a esa respuesta al cometer algún error de operatividad, razonamiento o visualización, en el caso de las preguntas de geometría. Sin embargo, al hacer el procedimiento adecuado se obtendrá la solución correcta de cada una de las preguntas.

El nivel de las preguntas que se plantea es el que debieron alcanzar, de acuerdo con los objetivos cognitivos que deben cumplirse en bachillerato y a las mallas existentes para el bachillerato unificado en el Ecuador, que abarcan contenidos desde aritmética, algebra, geometría y trigonometría. Todas las preguntas responden a un nivel medio de complejidad, aunque algunas podrían considerarse que son más sencillas por la rapidez con que se realizan las operaciones para hallar la solución correcta.

Un ejemplo de la complejidad de las preguntas lo podemos ver, colocando dos por cada área:

a. Indique el valor en radianes de 210

b. Si la ¿cuál es el valor de?

2. 3. ¿Cuál es el valor de c, para que la siguiente expresión sea un cuadrado perfecto? 
a. La ecuación que convierte grados Centígrados a grados Fahrenheit. ¿Cuál de las siguientes ecuaciones convierte de grados Fahrenheit a grados Centígrados?

3. 5. El área de un triángulo equilátero de lado $\mathrm{m}$, es:

4. 6. Los triángulos rectángulos $\triangle \mathrm{ABC}$ y $\triangle \mathrm{DEF}$ son semejantes. La hipotenusa de $\triangle \mathrm{ABC}$ mide $5 \mathrm{~cm}$ y la de $\triangle \mathrm{DEF}$ mide $10 \mathrm{~cm}$. El menor de los catetos de $\triangle \mathrm{ABC}$ mide $3 \mathrm{~cm}$. ¿Cuál es la longitud del mayor de los catetos de $\triangle \mathrm{DEF}$ ?

5. 7. El resultado de realizar las siguientes operaciones $4 \times 3-5 \times 2-3$ es:

6. 8. ¿Cuál de los siguientes números se encuentra entre $1 / 2$ y 3/5?

Las preguntas fueron variadas en cuanto a la dificultad, no colocando las sencillas primero y las de mayor dificultad después, sino que se alternaron para que el estudiante tuviese el tiempo necesario para razonar cada una de las respuestas y no se sintiera presionado por la dificultad de la pregunta. Esto se hizo para cada una de las áreas y subtemas.

\section{Conclusiones}

- Las áreas de mayor relevancia, indicadas por los expertos con experiencia en la enseñanza de matemática en diversas carreras de la Escuela Superior Politécnica de Chimborazo, para poder cumplir los objetivos planteados en el primer semestre de las carreras dictadas en la facultad de ciencias son: Aritmética, Algebra, Geometría y Trigonometría.

- El Nivel cognitivo de aprendizaje del instrumento es complejidad media y abarca los dominios relacionados con Aplicación y Análisis de acuerdo a la taxonomía de Bloom.

- El Instrumento elaborado está diseñado para responderse en 90 min, y está formado por 32 preguntas de los cuales ocho corresponden al área de Aritmética, doce de Algebra, seis de Geometría y seis de Trigonometría. Siendo Algebra la de mayor cantidad de preguntas, ya que los expertos indicaron una gran cantidad de subtemas en esa área indicándola como la que mayores necesidades tienen los estudiantes al iniciar una carrera de la Facultad de Ciencias.

- Los datos socio -demográficos se incorporan con el fin de establecer relaciones entre el nivel de conocimientos y la procedencia, edad, situación familiar de los estudiantes. Así como la correlación con las diferentes carreras, esperándose mayores conocimientos en los estudiantes procedentes de área urbanas que de áreas rurales o los procedentes de colegios privados que de colegios públicos. 


\section{Referencias bibliográficas}

Alemany, I., \& Lara, A. (2010). Las actitudes hacia las matemáticas en el alumnado de ESO: un instrumento para su medición. Publicaciones., 40, 49-71.

Alsina, A. \& Coronata, C. (2014). Los procesos matemáticos en las prácticas docentes: diseño, construcción y validación de un instrumento de evaluación. Edma 0-6: Educación Matemática en la Infancia., 3(2), 23-36.

Alvarado, E., Canales, F. \& Pineda, E. (1994). Metodología de la Investigación (2ª ed.). Washington D.C: Organización Panamericana de la Salud

Biggs, J. (2003) Teaching for quality learning at university. Assessing for learning quality: II. Practice", https://www.academia.edu/30307405/Teaching_for_Quality_Learning_at_University

Cerda, G., Pérez, C., Moreno, C., Núñez, K., Quezada, E., Sáez, S., \& Rebolledo, J. (2012). Adaptación de la versión española del test de evaluación matemática temprana de UTRECHT en Chile. Estudios Pedagógicos., 38(1), 235-253.

Corral, Y. (2009). Validez y confiabilidad de los instrumentos de investigación para la recolección de datos. Revista Ciencias de la Educación., 19(33), 228-247.

Darías, E. (2005). Cuestionario de actitudes hacia las matemáticas para alumnos de Tenerife. Comunicación presentada al II Congreso Hispano-Portugués de Psicología.

Gadermann, A., Guhn, M., \& Zumbo, B. (2012). Estimating ordinal reliability for Likerttype and ordinal item response data: A conceptual, empirical, and practical guide. Practical Assessment, Research \& Evaluation, 17(3), 1-13.

García, R. (2018). Diseño y construcción de un instrumento de evaluación de la competencia matemática: aplicabilidad práctica de un juicio de expertos. Ensaio Avaliação e Políticas Públicas em Educação., 26(99), 347-372.

Malhotra, N. (2004). Investigación de Mercados (4ta ed.). Pearson Education, México. 
Ministerio de Educación. Libro texto de Matemática 3er curso texto del estudiante. Recuperado de https://educacion.gob.ec/libros-de-texto/

Muñoz, J., \& Mato, M. (2006). Diseño y validación de un cuestionario para medir las actitudes hacia las matemáticas en alumnos de ESO. Revista Galego-portuguesa de Psicoloxia e Educación., 13(11-12), 413-424.

National Council of Teachers of Mathematics (2000). Principles and standards for school mathematics. Reston, Va.: The National Council of Teachers of Mathematics (Trad. Castellana, Principios y estándares para la educación matemática. Sevilla: Sociedad Andaluza de Educación Matemática Thales, 2003). http://standards.nctm.org/.

Obando P. (2009). Construcción y validación de una prueba para medir conocimientos matemáticos. Horizontes Pedagógicos, 11(1), 29-37.

Oviedo, H., \& Campo-Arias, A. (2005). Aproximación al uso del coeficiente alfa de Cronbach. Revista colombiana de psiquiatría, 34(4), 572-580.

Robles, F. Las 10 etnias del Ecuador con mayor población. Lugar de publicación: Lifeder.com. Recuperado de https://www.lifeder.com/etnias-del-ecuador/.

Vallejo, G. \& Escudero, J. (1999). Cuestionario para evaluar las actitudes de los estudiantes de ESO hacia las matemáticas. Aula Abierta., 74, 1-8.

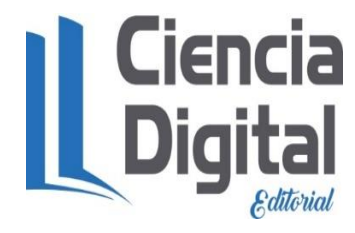




\section{PARA CITAR EL ARTÍCULO INDEXADO.}

Carreras García, F. de A., \& Sívoli Barrios, Z. M. (2020). Diseño y validación de un instrumento para medir los conocimientos en matemáticas básica que poseen los estudiantes de ciencias. Ciencia Digital, 4(1), 253-269. https://doi.org/10.33262/cienciadigital.v4i1.1098

\section{Ciencia}

El artículo que se publica es de exclusiva responsabilidad de los autores y no necesariamente reflejan el pensamiento de la Revista Ciencia Digital.

El artículo queda en propiedad de la revista y, por tanto, su publicación parcial y/o total en otro medio tiene que ser autorizado por el director de la Revista Ciencia Digital.
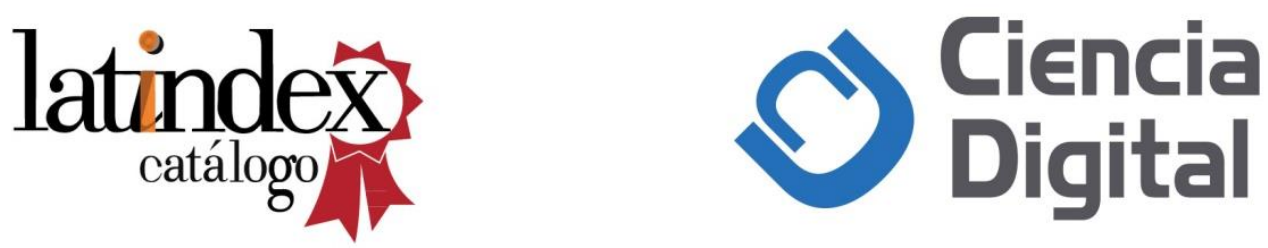